\title{
Politics matters. Dynamics of inter-organizational networks among immigrant associations
}

\author{
Matteo Gagliolo ${ }^{1,2}$, Tom Lenaerts ${ }^{2,3}$, and Dirk Jacobs ${ }^{1}$ \\ 1 GERME, Institute of Sociology \\ 2 MLG, Computer Science Department \\ Université libre de Bruxelles (ULB) \\ 3 AI Lab, Computer Science Department, Vrije Universiteit Brussel \\ Brussels, Belgium
}

\begin{abstract}
We model the dynamics of the two-mode network among directors and boards of voluntary associations, using a stochastic actorbased model, SIENA [12], including the structural effects proposed in [6], and considering the political orientation of associations as a covariate. Using data from [14], we compare the evolution of interlocks among Turkish associations in two European capitals, and explain the noticeable difference in structure by looking at statistically significant differences among the estimated effects.
\end{abstract}

\section{Introduction}

Social capital designs the ensemble of resources which are accessible to a social actor through its relationship with other actors [9]. As such, it is naturally embedded in social networks [1]. In his famous work on the causal relationship between "bridging" social capital (associational life), trust, and civic behavior, Putnam [10] did not investigate the structural aspect of such networks, leaving the question of its relevance unstated. Recently, the relationship between associational life and political participation of ethnic minority groups in Europe has been investigated, obtaining useful insights, yet without reaching uniform conclusions $[4,13,14]$. In this line of work, simple structural properties of the network of interlocking directorates among ethnic associations have been used as a "proxy" of the social capital of the corresponding minority group. The aim of our research is to pursue this line further, looking at the structure of such networks, but also at the dynamics that produce it. Here, we use a stochastic actor-based model, SIENA [12], which estimates the effect of actor covariates and local structure on network evolution, to analyze data from [14], describing the evolution of the councils of Turkish associations in Amsterdam and Berlin.

\section{Background}

The concept of social capital designs the ensemble of resources that an actor can access or mobilize via his connections to other actors, regardless of the kind of 
resource considered (information, control, support, etc.). Most of the early work on social capital focuses on individuals, or small elite groups [9]; its study at the aggregate level, for a whole community, has been popularized by Putnam [10], who observed a virtuous circle of causal connections between the amount of associational life, the level of trust, and civic behavior.

More recently, Fennema and Tillie [3] compared the social capital of four migrant communities in Amsterdam, studying structural differences among the networks of interlocking directorates of ethnic organizations (i.e., connections among organizations sharing one or more board members), and found a positive correlation among the number of interlocks in the network, and the political participation of the corresponding ethnic minority group. Later studies questioned this hypothesis, finding more subtle relationships among organizational network structures, aggregate indicators of immigrant groups, and civic behavior (see [5], and other papers from the same special issue).

Interlocking directorates [2] are an example of two-mode or bipartite networks, where two classes of nodes are present (in this case, directors and boards), and links are only possible from one class to the other. A projection onto two distinct one-mode networks can be performed, linking two nodes if they are connected to a same node of the other mode. This allows to draw a network among boards that have at least one director in common; or among directors that sit on the same board. As one mode networks have been the subject of a much larger corpus of research, and software development, many scholars prefer to analyze one of the projections, discarding the other mode. While not devoid of interest, this approach has several limitation, in that some of the information in the data is lost during the projection (e.g., in the case of boards, multiple common directors). Moreover, it has been shown that the projection introduces spurious structures in the one-mode network [7], changing the meaning of some of the standard network measures, such as density and clustering [8].

\section{Methods}

SIENA [12] is a stochastic actor-oriented model of network evolution, meaning that the structure of observed network data is assumed to be the result of the actions of a set of agents, each corresponding to a node, and exerting a control over its outgoing ties, by adding or deleting ties to the other nodes. Given a set of potential motives governing social choices, mathematically defined as effects, the algorithm estimates a set of parameters, each modulating the impact of the corresponding effect on the probability of forming and deleting ties. Based on a pair of snapshots, or waves, of an evolving network, the estimate is performed such that, in a simulation starting from the first snapshot, the final simulated snapshot will be the most similar to the one actually observed. In the following, we provide a simplified description of the model, based on [11] and [6].

Be $\mathbf{x} \in\{0,1\}^{N}$ the binary matrix representing a network among $N$ nodes, and $\Delta_{i j} \mathrm{x}$ the matrix obtained by switching a single element of $\mathbf{x},\left(x_{i j} \leftarrow 1-x_{i j}\right)$. Each node $i$ is an agent, which can perform atomic changes to its outgoing ties $x_{i j}$, 
at exponentially distributed points in time, with rate $\lambda$. Agents add and remove links according to a "perturbed" utility function: the target index $j$ is drawn with probability $p_{i}(j \mid \mathbf{x}) \propto \exp \Delta f_{i}(j, \mathbf{x})$, where $\Delta f_{i}(j, \mathbf{x})=f_{i}\left(\Delta_{i j} \mathbf{x}\right)-f_{i}(\mathbf{x})$ is the variation in utility that would be obtained by $i$ with a switch of $x_{i j}$.

The utility function $f_{i}(j, \mathbf{x})$ is a linear combination of effects, which can be arbitrary functions of the current network $\mathbf{x}$, as well as of node covariates:

$$
f_{i}(\mathbf{x})=\sum_{k} \theta_{k} s_{i, k}(\mathbf{x})
$$

Usually, the structural effects can be decomposed according to the outgoing ties of $i$, as $s_{i}(\mathbf{x})=\sum_{j} s_{i}(j, \mathbf{x})$. The model is Markovian: at each time step, the probability distribution over the possible next states (all networks at Hamming distance 1) can only depend on the current state. Fixing a set of effects and an initial network $\mathbf{x}$, its further evolution will be a stochastic function of the rate $\lambda$ and the effect weights $\boldsymbol{\theta}=\left\{\theta_{k}\right\}$. These parameters can be estimated with a Markov chain Monte Carlo approach, selecting those values which produce networks that are the most similar to the ones observed, in terms of the aggregate values of the included effects. The standard deviation of effect estimates can be used to test their significance with a simple $t$-test. Also differences among an effect estimated on distinct data sets can be tested for significance ${ }^{4}[11]$.

As previously pointed out (Sec. 2), analyzing the one mode projection of two-mode data can be misleading ${ }^{5}$. Luckily for us, the method has been recently extended to two-mode networks by [6], who applied it to model the evolution of interlocking directorates among firms in the Swedish stock market. In the paper, four structural effects were considered as relevant for interlock formation (Table 3): the basic outdegree effect, density; simple and double interlocks, labeled 2-star and 4-cycle, respectively; and an intermediate structure termed 3-path. The resulting four effects have distinctly different meanings: while density is a "baseline" expressing the tendency of associations of acquiring a new director ${ }^{6}$, 2-star corresponds to the tendency of forming an interlock, which may indicate an underlying social connection among the new director and members of the board, or reflect a strategy of the two boards; and 4-cycle amounts to adding a second interlock to an existing one, which is interpreted by [6] as a stronger clue of peer referral: the fact that at least one of the directors sits already on both boards implies that he knows already all directors involved, and may suggest that one of his colleagues from the first board joins the second one, thus doubling the

\footnotetext{
${ }^{4}$ Comparing different effects on the same data set is instead not trivial, as the magnitude of an effect is not a relevant index of its actual impact on network evolution, which also depends on the magnitude of the associated effect function. What matters in interpreting the results is the sign of significant effects.

${ }^{5}$ It is especially problematic with SIENA, as the basic assumption of atomicity of changes (agents can only change one link at a time) is violated. For example, by joining a new board, a director that sits already on $k$ boards will add $k$ new links at once in the projection, among the new board and the previous ones

${ }^{6}$ The mode of the organizations is considered the active one, unilaterally deciding when to add/remove directors.
} 


$$
\begin{aligned}
\text { density } & s_{i}(j)=x_{i, j} \\
\text { 2-star } & s_{i}(j)=x_{i, j} \sum_{h \neq i} x_{h, j} \\
\text { 3-path } & s_{i}(j)=x_{i, j} \sum_{h \neq i} x_{h, j} \sum_{k \neq j} x_{h, k} \\
\text { 4-cycle } & s_{i}(j)=x_{i, j} \sum_{h \neq i} x_{h, j} \sum_{k \neq j} x_{h, k} x_{i, k}
\end{aligned}
$$

Table 1. Structural effects used in [6] (argument $\mathbf{x}$ dropped to simplify the notation).

interlock. The 3-path is added for completeness, as an alternative explanation to 4-cycle formation, and may be interpreted as a differential preference for interlocks towards boards with a larger number of directors.

\section{Results}

The analysis was carried out using RSiena [11]: in the following we illustrate the details of the experiments, and the results obtained.

Data. Longitudinal data (1985-2003) of the composition of directorates of Turkish associations in Berlin and Amsterdam was provided by the authors of [14], along with a broad classification of the political orientation of the organizations, on three levels (left, center, right, which we encoded as -1, 0, 1).

Settings. Following [6], the boards mode was considered the active one, choosing which directors to recruit and release. The default Robbins-Monro approximation was used for estimating the parameters. The estimates were performed on a sequence of data sets, obtained using a shifting window of four consecutive waves (four years).

Effects. The same structural effects of [6] were used (see Table 3). In order to study the impact of political orientation on interlock formation, we implemented a distance 2 similarity effect ( $\operatorname{simD2}$ ), to measure the preference for interlocks with similar associations, corresponding to an homophily effect in the one-mode projection. simD2 was defined $a^{7}$ :

$$
s_{i}(j)=x_{i j} \frac{\sum_{h \neq i} x_{h j} \operatorname{sim}_{i h}}{\sum_{h \neq i} x_{h j}} .
$$

Estimates. In a first set of experiment (Fig. 4), we tested the four structural effect described above. While the numbers involved are much different (interlocks are a much rarer phenomenon in the voluntary sector), the results follow a similar pattern to that observed by [6] on much denser interlock networks in the for profit sector. More precisely, the density effect is negative, due to the limited number of directors per board, implying that the vast majority of possible ties are absent. The 2-star effect is mostly negative, suggesting that boards do

\footnotetext{
${ }^{7}$ Here, $v_{i}$ is the covariate value of $i, \Delta_{v}=\max _{i h}\left|v_{i}-v_{h}\right|$ the observed range of the covariate, and $\operatorname{sim}_{i h}=\frac{\Delta_{v}-\left|v_{i}-v_{h}\right|}{\Delta_{v}}$ the similarity among two nodes $i$ and $h$ in the same mode. Therefore, simD2 varies between 0 (for interlocks connecting two boards with maximum covariate difference) and 1 (for identical covariates).
} 


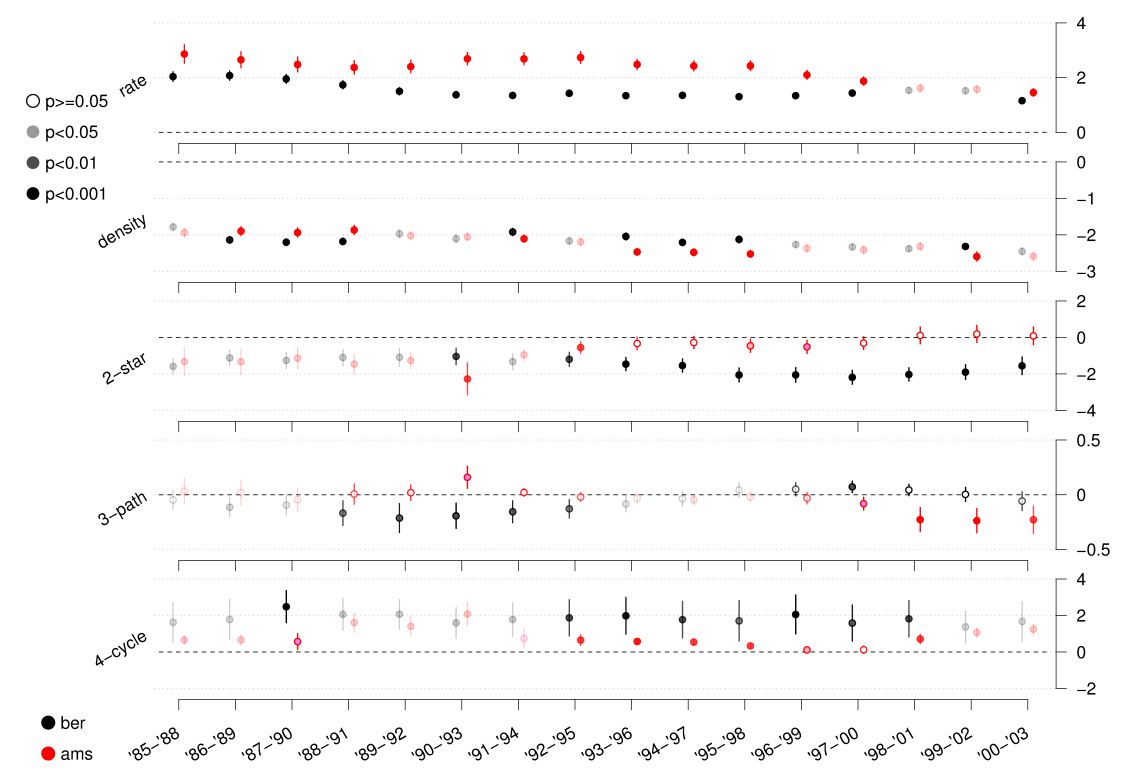

Fig. 1. SIENA estimates, structural effects only. Each horizontal section corresponds to an effect. Ticks on the horizontal axes correspond to four consecutive years of data. For each effect, at each year, two circles are plotted, indicating the estimated values for Berlin (left, black), and Amsterdam (right, red). Full circles indicate significant results (based on $p$-values, see legend at upper left), while empty circles are not significant. Vertical segments represent the standard deviation of each estimate. Significant difference among the two towns is instead highlighted using a transparent tint effect where the difference is not significant.

not actively search to form interlocks: note that, given that density acts as a baseline, a negative 2-star means that associations prefer to enroll an inactive director (forming a simple tie) rather than someone who is already active in another board (forming an interlock). The 3-path is often not significant, and when it is, its value is quite small (i.e., when forming an interlock with another board, its size does not matter): in our case, this effect is small but significantly negative in Berlin for some of the waves (1986-1995 and 1997-2003), indicating a moderate preference for smaller boards. This needs further interpretation: it may be related to the existence in Berlin of so-called umbrella organizations, which aim at coordinating the activities of several smaller ones, enrolling one director for each member association. 4-cycle is, instead, significantly positive. In practice this means that, when a simple interlock is already present, an association will prefer to enroll a director from the connected board, rather than a complete outsider. In [6], this is interpreted as a clue of peer referral. 


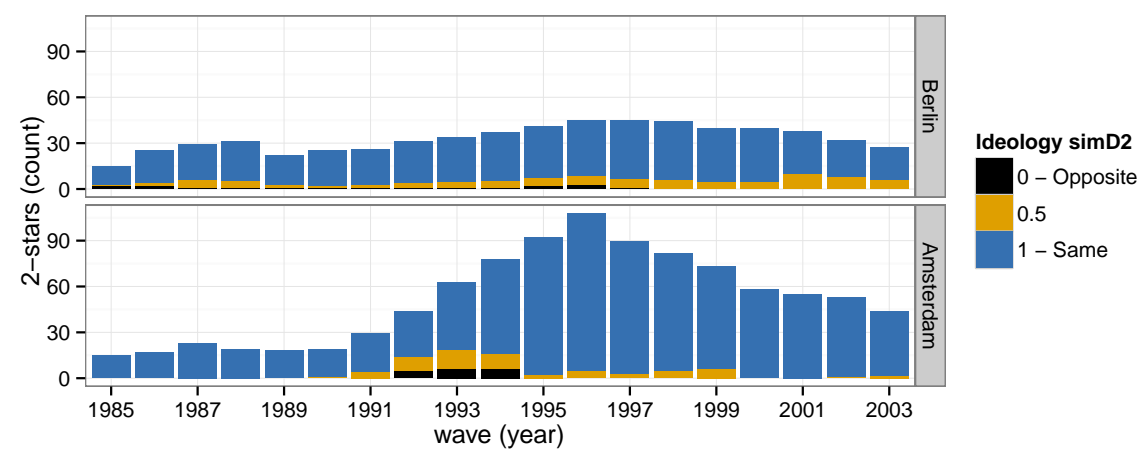

Fig. 2. Simple interlocks (2-star), per year, in Amsterdam and Berlin. Colors indicate the ideology similarity among the two interlocked organizations (0: left-right; 0.5 : center-left, center-right; 1 left-left, right-right).

While we cannot compare the magnitudes of different effects on a given data set (see note 4), we can however compare the estimates of the same effect on different data sets: in particular, we can check for statistically significant differences among the two data-sets. In this case, the first obvious difference is in the rate parameter, which is almost always higher in Amsterdam, indicating more frequent variations in the composition of the boards. In terms of the structural effects, a comparison among Amsterdam and Berlin reveals that Turkish associations in Berlin have a stronger negative 2-star and stronger positive 4-cycle, suggesting that they form interlocks less easy, but are more prone to reinforcing them when present, compared to Turks in Amsterdam: however, the absolute number of four cycles in Berlin is very small (hence the large standard deviations of the estimates).

This difference is reflected in the evolution of these two indicators in the two towns (see Fig. 2 for 2-star). While similar during the 1980s, both indicators start diverging during the early 1990s: the steeper increase of interlocks in Amsterdam corresponds to a significant difference in the 2-star effect during this decade. Grouping interlocks according to the similarity of the connected associations, we can remark that political polarization is very strong in both cities, as the vast majority of interlocks connect associations from the same political side.

Are these differences sufficient to explain the differences in structure observed in the two cities? Intuitively, politics must play a role, otherwise the interlocks would not follow political alignment. After implementing the simD2 effect, we could test the impact of similarity among associations, including also a net effect of political orientation (Fig. 3). While density and 2-star remain unaffected, the 4-cycle effect keeps the same pattern, but its difference looses significance in most of the waves, confirming that structural effects alone are not sufficient to explain this data: as the vast majority of four cycles links politically homogeneous organizations, adding this covariate effect renders the structural one su- 


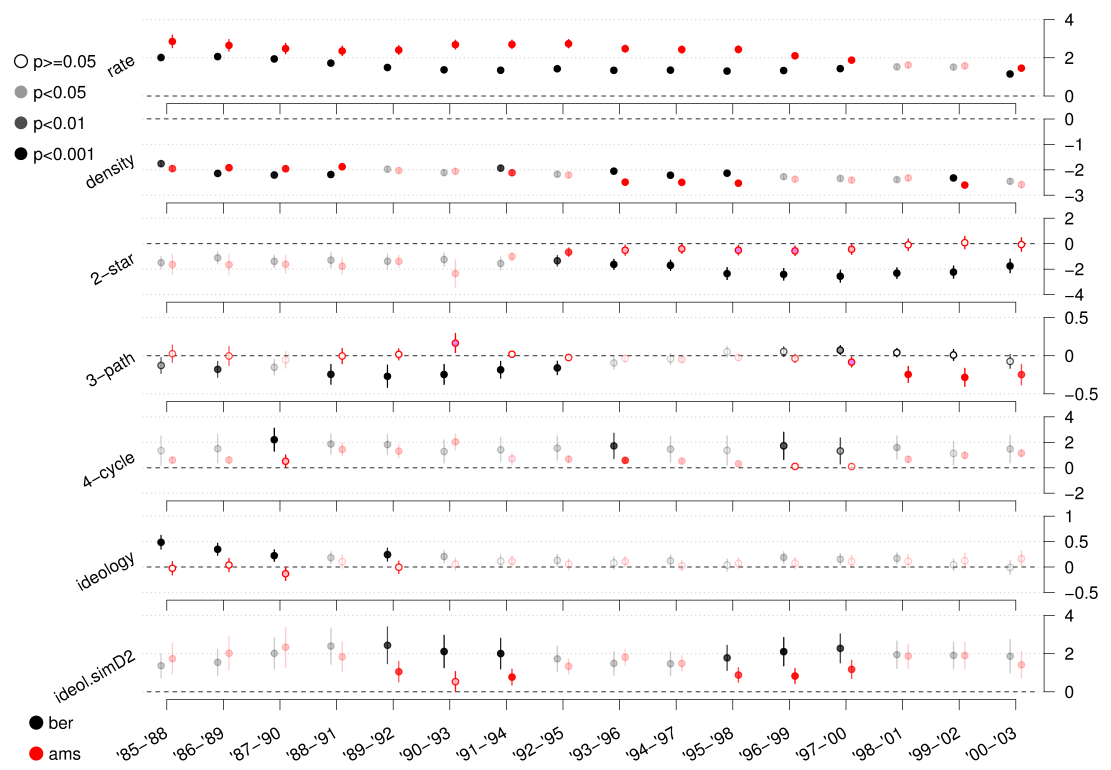

Fig. 3. SIENA estimates, including covariates. See Figure 4 for the legend.

perfluous. Ideology by itself is also mostly not significant, except in Berlin during 1985-1992, indicating a greater activity of right-wing associations in recruiting directors. The political affinity among different associations (ideol.simD2) is instead relevant. It is mostly significant, and positive, in both towns. Regarding significant differences, we can identify two periods where the similarity effect is significantly lower in Amsterdam, in the early and late 90's. This corresponds to periods characterized by a slight increase of politically heterogeneous interlocks in this town (see Figure 2).

\section{Conclusions}

While preliminary, our results already highlight the importance of political homophily in interlock formation, and allow to describe the difference among the two communities in quantitative terms. In this sense, the 2-star effect seems particularly relevant. In the longer term, we intend to relate the dynamics of these networks to the political participation of the corresponding communities, in order to test existing hypotheses on the impact of ethnic social capital on political participation [3,4].

Acknowledgments. We would like to thank Floris Vermeulen and Jean Tillie (Political Science Dept., University of Amsterdam) for kindly contributing 
the data on Amsterdam and Berlin; and Johan Koskinen for useful insights on his work. The first author is supported by the Brussels Institute for Research and Innovation (Innoviris), under the program "Prospective Research for Brussels".

\section{References}

1. Burt, R.: The network structure of social capital. Research in Organizational Behavior 22, 345-423 (2000)

2. Fennema, M., Schijf, H.: Analysing interlocking directorates: Theory and methods. Social Networks 1(4), 297-332 (1978), http://dx.doi.org/10.1016/ 0378-8733 (78) 90002-3

3. Fennema, M., Tillie, J.: Civic community, political participation and political trust of ethnic groups. Connections 24(1), 26-41 (2001), http://www.insna.org/PDF/ Connections/v24/2001_I-1_26-41.pdf

4. Jacobs, D., Phalet, K., Swyngedouw, M.: Associational membership and political involvement among ethnic minority groups in brussels. Journal of Ethnic and Migration Studies 30(3), 543-559 (2004), http://dx.doi.org/10.1080/ 13691830410001682089

5. Jacobs, D., Tillie, J.: Introduction: Social capital and political integration of migrants. Journal of Ethnic and Migration Studies 30(3), 419-427 (2004)

6. Koskinen, J., Edling, C.: Modelling the evolution of a bipartite network - peer referral in interlocking directorates. Social Networks 34, 309-322 (2012), http: //dx.doi.org/10.1016/j.socnet.2010.03.001

7. Newman, M.E.J., Strogatz, S.H., Watts, D.J.: Random graphs with arbitrary degree distributions and their applications. Physical Review E 64(2), 026118 (2001)

8. Opsahl, T.: Triadic closure in two-mode networks: Redefining the global and local clustering coefficients. Social Networks 35(2), 159 - 167 (2013), http://dx.doi. org/10.1016/j.socnet.2011.07.001

9. Portes, A.: Social capital: Its origins and applications in modern sociology. Annual Review of Sociology 24(1), 1-24 (1998), http://dx.doi.org/10.1146/annurev. soc.24.1.1

10. Putnam, R.: Making democracy work : civic traditions in modern Italy. Princeton University Press, Princeton, N.J (1993)

11. Ripley, R.M., Snijders, T.A., Preciado, P.: Manual for siena version 4.0 (version january 17, 2012). Tech. rep., Oxford: University of Oxford, Department of Statistics; Nuffield College. (January 2012), http://www.stats.ox.ac.uk/siena/

12. Snijders, T.A.B., van de Bunt, G.G., Steglich, C.E.G.: Introduction to stochastic actor-based models for network dynamics. Social Networks 32(1), 44-60 (2010), http://dx.doi.org/10.1016/j.socnet.2009.02.004

13. Tillie, J.: Social capital of organisations and their members: Explaining the political integration of immigrants in amsterdam. Journal of Ethnic and Migration Studies $30(3), 529-542(2004)$

14. Vermeulen, F., Berger, M.: Civic networks and political behavior: Turks in amsterdam and berlin. In: Ramakrishnan, S.K., Bloemraad, I. (eds.) Civic Hopes and Political Realities: Immigrants, Community Organizations and Political Engagement, pp. 160-192. Russell Sage Foundation Press, New York (2008) 